\title{
Zur Bewertung von Entscheidungsfindungen
}

\section{Kommentar zum Beitrag „Führen und Entscheiden in polizeilichen Extremsituationen“von Ralf Martin Meyer}

Der Polizeipräsident hat in seinem Beitrag den Fokus auf den Weg der Entscheidungsfindung, die Beratungskultur und die nachträgliche Reflexion sowie die Verantwortungsübernahme gelegt. Es ging ihm dabei primär um die internen Abläufe beim polizeilichen Krisenmanagement. Die rechtswissenschaftliche Perspektive und auch die juristische Praxis rücken demgegenüber klassischerweise die nachträgliche, vor allem gerichtliche Kontrolle in den Mittelpunkt. Der Entscheidungsprozess selbst wird dabei typischerweise eher indirekt, abgeleitet reflektiert - auch deshalb, weil dem Theoretiker zu verwaltungs- und polizeiinternen Abläufen regelmäßig der Zugang und das unmittelbare Anschauungsmaterial fehlen. Allerdings hat es sich seit einigen Jahren die sog. „Neue Verwaltungsrechtswissenschaft“ [1,2] auf die Fahne geschrieben, die Handlungs- und Bewirkungsperspektive des Verwaltungsrechts (wozu auch das Polizei- und Ordnungsrecht gehört) stärker in den Blick zu nehmen.

Für das polizeiliche Handeln in Extremsituationen bedarf es rechtlicher Mechanismen, um einerseits eine informierte und rationale Entscheidungsfindung sowie die Beachtung der Grundrechte der Betroffenen zu gewährleisten und andererseits die Entscheidungsträger gerade in Extremsituationen nicht mit unrealistisch überzogenen Anforderungen zu überfrachten. In der Sache geht es zentral um die Frage, welche rechtlichen Anforderungen an Prognosen zu stellen sind, wenn es gilt, die Konsequenzen verschiedener Handlungsoptionen $\mathrm{zu}$ bewerten und $\mathrm{zu}$ vergleichen. Aus der Kontrollperspektive kommen die Fragen nach Kontrollmaßstab und Kontrolldichte hinzu. Insbesondere fragt sich, ob bei der Gerichtskontrolle nachträgliche Erkenntnisse noch berücksichtigt werden können und inwieweit den handelnden Akteuren Spielräume einzuräumen sind.

\section{Unterscheidung zwischen Gefahr und Risiko}

Rechtsdogmatisch grundlegend erscheint zunächst einmal die Unterscheidung zwischen Gefahr (mit der klassischen Polizeiaufgabe der Gefahrenabwehr) und bloßem Risiko (mit eventueller Notwendigkeit der Gefahrenvorsorge) [3,4]. Diese Differenzierung ähnelt der in der Ökonomie [5] und anderen Wissenschaften [6] verbreiteten Unterscheidung von Risiko (gleich juristisch Gefahr) und Unsicherheit (gleich juristisch Risiko) [7]. Bei der Gefahr sind definitionsgemäß Szenarien und Eintrittswahrscheinlichkeiten im Wesentlichen (ggf. mit einer prozentualen Marge) bekannt, sodass sich jedes Szenario theoretisch in einem Produkt aus Resultat und Eintrittswahrscheinlichkeit bewerten und vergleichbar machen lässt. Selbstverständlich sind sich auch 
Juristen dessen bewusst, dass bei menschlichem Verhalten Wahrscheinlichkeiten im streng mathematischen Sinne jedenfalls dort, wo nur relativ wenige Erfahrungswerte existieren, nicht exakt berechnet werden können. Man begnügt sich mit einer annäherungsweisen Schätzung, gepaart mit einer normativ-wertenden Betrachtung [8], wie sie zur Bestimmung einer „überwiegenden“, „,hohen“ oder gar „mit an Sicherheit grenzenden“ Wahrscheinlichkeit notwendig ist. Die Kategorie des Risikos soll demgegenüber Situationen erfassen, ,die sich durch Unsicherheiten über Schadensmöglichkeiten und Eintrittswahrscheinlichkeiten sowie über die Kausalität von Ursache und Wirkung auszeichnen“ [9,10]. Allerdings wird diese kategoriale Unterscheidung von Gefahr und Risiko keineswegs von allen geteilt. Es gibt auch Stimmen, die angesichts gleitender Übergänge das Risiko als Oberbegriff sehen [11,12]. Die Rechtsprechung geht zwar von der obigen Unterscheidung aus, doch verfließen die Grenzen zwischen Gefahr und Risiko teilweise wieder, wenn das Bundesverfassungsgericht und andere auch beim Risiko die „Je-desto-Formel“ bemühen. Für die Annahme eines rechtlich relevanten Risikos (in Abgrenzung zum hinzunehmenden bloßen „Restrisiko jenseits praktischer Vernunft“) genügt nämlich eine umso geringere prozentuale Wahrscheinlichkeit des Schadenseintritts, je größer der bei Untätigkeit zu erwartende Schaden zu werden droht [13,14] - was logisch eine zumindest annähernde Bestimmbarkeit von Wahrscheinlichkeiten voraussetzt. Diese „Je-desto-Formel“ ist freilich nicht streng mathematisch zu verstehen; vielmehr mischen sich einmal mehr normativ-wertende Elemente (,jenseits praktischer Vernunft“ versus hinreichende Wahrscheinlichkeit zur Überschreitung der Relevanzschwelle) mit Rechenoperationen (Ermittlung eines Produkts von idealiter prozentualer Wahrscheinlichkeit eines Schadens und Schadenshöhe, wobei freilich die Schadenshöhe bei immateriellen Schäden wiederum ein Stück weit wertend bestimmt werden muss).

Traditionell werden auch die von Herrn Meyer geschilderten polizeilichen Extremsituationen - im Gegensatz zu manchen Szenarien bei der vorsorgenden Kriminalitätsbekämpfung, insbesondere der Datenverarbeitung im Vorfeldbereich [15] - in diesem Sinne der Gefahrenabwehr und nicht dem Risiko und der Gefahrenvorsorge zugerechnet [16]. Daran lässt sich jedoch mit Fug und Recht zweifeln, wenn man den Zeitdruck mitberücksichtigt. Denn dieser kann eine fundierte Wahrscheinlichkeitsabschätzung hinsichtlich weiterer Szenarien unmöglich machen. Bei bereits begonnenen terroristischen Attacken oder Amokläufen steht die höchste Gefahrenstufe als solche außer Zweifel, doch weiß man nicht unbedingt, wohin sich der oder die Täter wenden werden. Bei Geiselnahmen ist der Grad der Wahrscheinlichkeit unsicher, mit dem Leben und Gesundheit des Opfers bei verschiedenen polizeilichen Reaktionen gefährdet sind. 


\section{Entscheidungsfindung}

Auch die Rechtswissenschaft geht vom Leitbild einer rationalen und das heißt im Normalfall möglichst informierten synoptischen Entscheidungsfindung aus. Dies allerdings auf einer recht pragmatischen Ebene, ohne sich auf ein bestimmtes Menschenbild (homo oeconomicus oder verhaltensökonomische Rationalitätsskepsis [17,18]) festzulegen. Gute Ausbildung und sachverständige Beratung der Entscheidungsträger sollen dies unterstützen. Insoweit kann ich die Aussagen von Herrn Meyer nur unterstreichen.

Weniger diskutiert ist, inwieweit das Idealbild der informierten, verschiedene Szenarien vergleichenden Berechnung gerade unter Zeitdruck und dadurch bedingter Informationsdefizite durch Heuristiken, durch Intuition und „Daumenregeln“ ergänzt oder modifiziert werden kann oder muss. Ich habe in etwas anderem Zusammenhang unter Berufung nicht zuletzt auf Gigerenzer [6] dafür plädiert [7]. Allerdings muss Intuition aus Erfahrung wachsen und darf nicht zur Chiffre für Beliebigkeit oder gar Vorurteile werden. Die vorschnelle Fixierung auf die „üblichen Verdächtigen“ (z. B. Migranten, „linke Chaoten“ usw.) ist auch bei politischem Erfolgsdruck keine rechtsstaatliche Option. Um dies zu verhindern, zeigt sich das Recht in vielen Vorschriften eher skeptisch gegenüber einem undifferenzierten Rekurs auf polizeiliche Erfahrung und verlangt eine (klare) Tatsachenbasis für die Prognose.

\section{Erkenntnisstand}

Unsicherheit und Zeitdruck werden vor allem dadurch berücksichtigt, dass man bei der späteren (Gerichts-)Kontrolle auf den Erkenntnisstand ex ante, also im Zeitpunkt der Entscheidungsfindung, und nicht ex post (zum Zeitpunkt der gerichtlichen Kontrolle) abstellt [19]. Hinterher ist man nämlich (fast) immer klüger. Maßgeblich ist das, was ein gut ausgebildeter und fähiger Polizeibeamter - das ist weniger als der „ideale Beamte“ - in der jeweiligen Situation erkennen und verarbeiten konnte und musste [20]. Indem so die juristischen Anforderungen auf ein gleichsam menschliches Maß reduziert werden, soll zugleich der psychologischen Tendenz entgegengewirkt werden, dass die Beamten aus Furcht vor rechtlichen Konsequenzen vor klaren Entscheidungen zurückschrecken.

\section{Beurteilungsspielraum}

Davon abgesehen billigt das Polizei- und Ordnungsrecht den handelnden Akteuren auf Polizeiebene keinen Beurteilungsspielraum bei der Einschätzung zu, wo die Gefahrenschwelle - definiert als hinreichende Wahrscheinlichkeit eines Schadenseintritts - überschritten ist. Insoweit bleibt ihre Einschätzung unter der Prämisse der ex-ante-Perspektive idealiter gerichtlich voll überprüfbar [21]. De facto wirkt sich der Entscheidungsmaßstab ex ante aber teilweise ähnlich kontrollreduzierend aus, wie es bei einem gerichtlich nur eingeschränkt überprüfbaren Beurteilungsspielraum der Fall wäre. 
Zudem gibt es auf der Rechtsfolgenseite im Polizeirecht typischerweise Ermessen. Als Auswahlermessen wird es gerade in den von Herrn Meyer skizzierten Szenarien besonders bedeutsam, in denen verschiedene Handlungsoptionen existieren. Streng genommen kommt dieses Ermessen als nur eingeschränkt (auf Ermessensfehler in Form von Ermessensnichtgebrauch, -fehlgebrauch und -überschreitung [22]) überprüfbarer Handlungsspielraum jedoch erst ins Spiel, nachdem schon die Prognosen richtig getroffen worden sind. Ob sich dies wirklich immer so klar trennen lässt, muss freilich bezweifelt werden.

\section{Einschätzungsspielraum}

Auf Regierungsebene räumt die Verfassungsrechtsprechung seit der Schleyer-Entscheidung [23] dagegen den politisch Verantwortlichen darüber hinaus bei der Erfüllung ihrer grundrechtlichen Schutzpflichten für die Bürger einen recht weiten Einschätzungsspielraum ein. Denn die Betreffenden müssen sich primär demokratisch bei den nächsten Wahlen und nicht juristisch vor Gericht (Gewaltenteilung!) verantworten. Je weitreichender die potentiellen Folgen, umso größer tendenziell der Spielraum. Die äußerste Grenze bleibt die unantastbare Menschenwürde, wie das Bundesverfassungsgericht beim Verbot des präventiven Abschusses eines entführten Passagierflugzeugs (Luftsicherheitsgesetz) noch einmal verdeutlicht hat [24].

\section{Prozeduralisierungsstrategie}

Wenn sich Entscheidungen nicht im Voraus in ihrem Inhalt gesetzlich determinieren lassen, wie dies hier typischerweise aufgrund ihrer Einzelfallabhängigkeit der Fall ist, sucht das Recht die Entscheidung prozedural zu steuern [25]. Eine solche „Flucht ins Verfahren“ im engeren Sinne scheidet in unseren Konstellationen freilich oftmals aus, weil unter Zeitdruck kein Raum für elaborierte Verwaltungsverfahren bleibt. Im weiteren Sinne lassen sich jedoch auch eine gute Vorbereitung durch Organisation, Planung und Strukturierung von Entscheidungsabläufen als eine solche Prozeduralisierungsstrategie einordnen. Denn derartige Vorkehrungen, deren Notwendigkeit Herr Meyer gerade zur Vorbereitung auf Terrorattacken betont hat, sollen juristisch dafür kompensieren, dass das (Polizei-)Recht in der Sache für Strategien und Einsätze in Extremsituationen nur grobe „Leitplanken“ liefern, aber keine genauen inhaltlichen Vorgaben machen kann.

Allgemein setzt das (Verfassungs-)Recht bei Entscheidungen unter Unsicherheit oftmals auf Beobachtungs- und Nachbesserungspflichten [26]. Nachträgliche Korrekturen dürften in den hier thematisierten Extremsituationen indes kaum möglich sein. Umso wichtiger werden aber nachträgliche Evaluationen, um es beim nächsten Mal gegebenenfalls besser zu machen (soweit die Situationen überhaupt vergleichbar sind). Auch insoweit kann ich den Ausführungen des Herrn Polizeipräsidenten aus rechtswissenschaftlicher Perspektive beipflichten. 


\section{Literatur}

[1] Voßkuhle A. Neue Verwaltungsrechtswissenschaft. In: Hoffmann-Riem W, Schmidt-Aßmann E, Voßkuhle A (Hrsg.). Grundlagen des Verwaltungsrechts, Band 1, 2. Aufl. München: Beck, $2012, \S 1$.

[2] Fehling M. Die „neue Verwaltungsrechtswissenschaft“- Problem oder Lösung. In: Burgi M, (Hrsg.). Zur Lage der Verwaltungsrechtswissenschaft. Die Verwaltung, Beiheft 12, Berlin: Duncker \& Humblot, 2017. 65-103.

[3] Pieroth B, Schlink B, Kniesel M. Polizei- und Ordnungsrecht. 9. Aufl. München: Beck, 2016: §4 Rn. $6 \mathrm{f}$.

[4] Jaeckel L. Gefahrenabwehrrecht und Risikodogmatik. Tübingen: Mohr Siebeck. 2010. 88 ff.

[5] Knight F. Risk, Uncertainty and Profit. Reprint New York: Kelly, 1964[1921]. 19 f., 197 ff.

[6] Mousavi S, Gigerenzer G. Risk, Uncertainty and Heuristics. In: Journal of Business Research. 2014;67:1671-1678.

[7] Fehling M. Der Umgang mit Unsicherheit in der ökonomischen Analyse des (Öffentlichen) Rechts. In: Hill H, Schliesky U (Hrsg.). Management von Unsicherheit und Nichtwissen. BadenBaden: Nomos, 2016. 203-237.

[8] Schenke W-R. Polizei- und Ordnungsrecht. 9. Aufl. Heidelberg: C.F. Müller, 2016. Rn. 77.

[9] Steinberg R. Der ökologische Verfassungsstaat. Frankfurt am Main: Suhrkamp, 1998. 21

[10] Di Fabio U. Entscheidungsprobleme der Risikoverwaltung. In: Natur und Recht. 1991;13(8):353359 (354, 357).

[11] Spiecker gen. Döhmann I. Rechtliche Strategien und Vorgaben zur Bewertung von Nichtwissen. In: Hill H, Schliesky U (Hrsg.). Management von Unsicherheit und Nichtwissen. Baden-Baden: Nomos, 2016. 89-112.

[12] Ladeur K-H. Das Umweltrecht der Wissensgesellschaft. Berlin: Duncker \& Humblot, 1995. $69 \mathrm{ff}$.

[13] Bundesverfassungsgericht, Amtliche Entscheidungssammlung (BVerfGE) 49. 89-147 (138) - Kalkar.

[14] Wahl R, Appel I. Prävention und Vorsorge. Von der Staatsaufgabe zur rechtlichen Ausgestaltung. In: Wahl R (Hrsg.). Prävention und Vorsorge. Bonn: Economica-Verlag, 1995.1-216 (4 ff.).

[15] Poscher R. Eingriffsschwellen im Recht der inneren Sicherheit. In: Die Verwaltung. 2008;41:345-373.

[16] Möstl M. Die staatliche Garantie für die öffentliche Sicherheit und Ordnung. Tübingen: Mohr Siebeck, 2002.192-198

[17] Engel C (Hrsg.). Recht und Verhalten, Tübingen: Mohr Siebeck, 2007.

[18] Fehling M. Ökonomische Analyse im Öffentlichen Recht als Methode zur Reformulierung und Operationalisierung von Gerechtigkeitsfragen. In: Die Fakultät der Bucerius Law School (Hrsg.). Begegnungen im Recht. Tübingen: Mohr Siebeck, 2011. 39-67 (52 ff.).

[19] Schenke W-R. Polizei- und Ordnungsrecht. 9. Aufl. Heidelberg: C.F. Müller, 2016. Rn. 69, 80.

[20] Pieroth B, Schlink B, Kniesel M. Polizei- und Ordnungsrecht. 9. Aufl. München: Beck, 2016. §4 Rn. 47.

[21] Schenke W-R. Polizei- und Ordnungsrecht. 9. Aufl. Heidelberg: C.F. Müller, 2016. Rn. $51,77$.

[22] Maurer H, Waldhoff C. Allgemeines Verwaltungsrecht. 19. Aufl. München: Beck, 2017. §9 Rn. 19-23.

[23] BVerfGE 46.160-165.

[24] BVerfGE 115.118-166 (153 f.)

[25] Hoffmann-Riem W. Eigenständigkeit der Verwaltung. In: Grundlagen des Verwaltungsrechts (siehe [1]). §10 Rn. 100.

[26] Fehling M. Der Umgang mit Unsicherheit in der ökonomischen Analyse des (Öffentlichen) Rechts (siehe [7]). $235 \mathrm{f}$. 